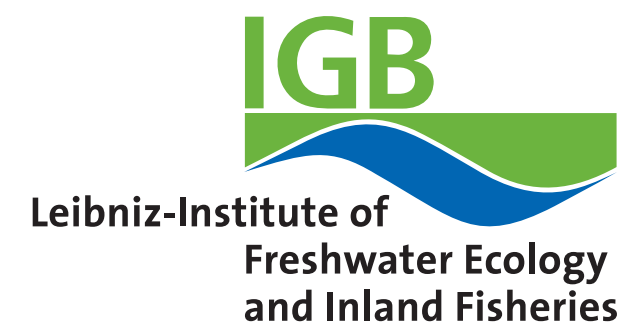

\title{
Experimental duration and predator satiation levels systematically affect functional response parameters
}

Yuanheng Li (D) https://orcid.org/0000-0003-4071-8212, Björn C. Rall (D) https://orcid. org/0000-0002-3191-8389, Gregor Kalinkat (iD https://orcid.org/0000-0003-3529-5681

DOI

https://doi.org/10.1111/oik.04479

Original publication date

25 October 2017 (Version of record online)

Document version

Accepted version

\section{Published in}

Oikos

\section{Citation}

Li Y, Rall BC, Kalinkat G. Experimental duration and predator satiation levels systematically affect functional response parameters. Oikos. 2018;127(4):590-8. 


\title{
Experimental duration and predator satiation levels systematically affect functional response parameters
}

\author{
Yuanheng Li ${ }^{* 1,2,3}$, Björn C. Rall ${ }^{\dagger 1,2}$, and Gregor Kalinkat ${ }^{\ddagger 4}$ \\ ${ }^{1}$ German Centre for Integrative Biodiversity Research (iDiv) \\ Halle-Jena-Leipzig, Deutscher Platz 5e, 04103 Leipzig, Germany \\ ${ }^{2}$ Institute of Ecology, Friedrich Schiller University Jena, Dornburger-Str. \\ 159, 07743 Jena, Germany \\ ${ }^{3}$ Johann-Friedrich-Blumenbach Institute of Zoology and Anthropology, \\ Georg-August-Universität Göttingen, Berliner Str. 28, 37073 Göttingen, \\ Germany \\ ${ }^{4}$ Department of Biology and Ecology of Fishes, Leibniz-Institute of \\ Freshwater Ecology and Inland Fisheries, Müggelseedamm 310, 12587 \\ Berlin, Germany
}

October 16, 2017

${ }^{\ddagger}$ kalinkat@igb-berlin.de; ORCID: http://orcid.org/0000-0003-3529-5681 
Empirical feeding studies where density-dependent consumption rates are fitted to functional response models are often used to parameterize the interaction strengths in models of population or food-web dynamics. However, the relationship between functional response parameter estimates from short-term feeding studies and realworld, long-term, trophic interaction strengths remains largely unexamined. In a critical first step to address this void, we tested for systematic effects of experimental duration and predator satiation on the estimate of functional response parameters, namely attack rate and handling time. Analyzing a large data set covering a wide range of predator taxa and body masses we show that attack rates decrease with increasing experimental duration, and that handling times of starved predators are consistently shorter than those of satiated predators. Therefore, both the experimental duration and the predator satiation level have a strong and systematic impact on the predictions of population dynamics and food-web stability. Our study highlights potential pitfalls at the intersection of empirical and theoretical applications of functional responses. We conclude our study with some practical suggestions for how these implications should be addressed in the future to improve predictive abilities and realism in models of predator-prey interactions.
\end{abstract}

Keywords: Type II functional response — handling time — attack rate — experimental duration — predator satiation level — diurnal cycle — digestive limit 


\section{${ }_{36}$ Introduction}

Understanding species interactions and how they shape communities and ecosystems is a core topic in ecological research. Trophic interactions are fundamental for ecosystems, as they determine energy flow and nutrient cycling in ecological networks (Elton, 1927; Brown et al., 2004; Thompson et al., 2012). Moreover, interaction strengths play a crucial role in determining population dynamics and stability of food webs (May, 1972, Oaten and Murdoch, 1975; Oksanen et al., 1981; Rall et al., 2008; Brose, 2010; Kalinkat et al., 2013; Li et al., 2017). Functional response models which describe per capita feeding rates of consumers as a function of resource densities (Solomon, 1949; Holling, 1959b) provide a widely applied and standardized way to quantify these interaction strengths in food webs (Berlow et al., 2004, Kalinkat et al., 2013). Accordingly, interaction strengths are typically quantified by empirical studies, carried out mostly in the laboratory, from which feeding data is collected and used to fit a functional response model Jeschke et al., 2002, 2004, Rall et al., 2012). Parameters from these models can then be used to parameterize the interaction strengths in theoretical food web models. Hence, functional response models often serve as the connection between studies of short-term, individuallevel interactions and long-term, community-level studies (e.g. Kalinkat et al., 2013). However, most functional response studies only investigate feeding over a short portion of a species lifetime, from minutes (e.g. Schröder et al., 2016) to a few days (e.g. Buckel and Stoner, 2000), and the results are often applied to studies modeling interactions over many generations (e.g. hundreds of years; Fox and Murdoch, 1978). Functional responses quantify the feeding rate of a predator averaging over the experimental duration, which is important for predicting numerical response (how predator density changes as a function of prey density over time) using, e.g. mathematical models (Okuyama, 2013). The combination of functional response and numerical response results in total response, 
the rate of overall prey population consumed by predator population (Holling, 1959a). Therefore, in community level studies the feeding rates have to represent the average consumption situation over the lifetime of a predator or many generations, not the feeding rates from a feeding trail that only includes a feeding event when the predator is hungry. Whether functional response parameter values derived from short-term functional response experiments hold for longer periods (e.g. on ecological time scales relevant to population dynamics) remains largely unexamined (but see Fox and Murdoch, 1978).

In a similar vein, the satiation levels of predators prior to feeding studies also may modify functional response parameter estimates. As a predator's satiation level directly affects its motivation to forage (Jeschke, 2007), satiated predators are expected to consume fewer prey individuals than starved predators which in turn would alter the functional response parameters. We addressed whether and how the experimental duration and the satiation level of predators affects the estimates of functional response parameters using a literature based functional response data base (Rall et al., 2012).

Due to the availability of data we focus our analysis on type II functional responses as described by Holling's disc equation (Holling, 1959b). This is the most widely-applied functional response model (Jeschke et al., 2002, 2004; Kalinkat and Rall, 2015), where the per capita feeding rate, $f(N)$, is formulated as a function of prey density, $N$ with two parameters, instantaneous rate of searching for prey, $a$ (hereafter: attack rate) and handling time, $h$ :

$$
f(N)=\frac{a N}{1+a h N}
$$

In this model, Holling (1959b) assumed that a predator spends its whole time budget on foraging, which includes activities such as searching, capturing, subduing, ingesting and 


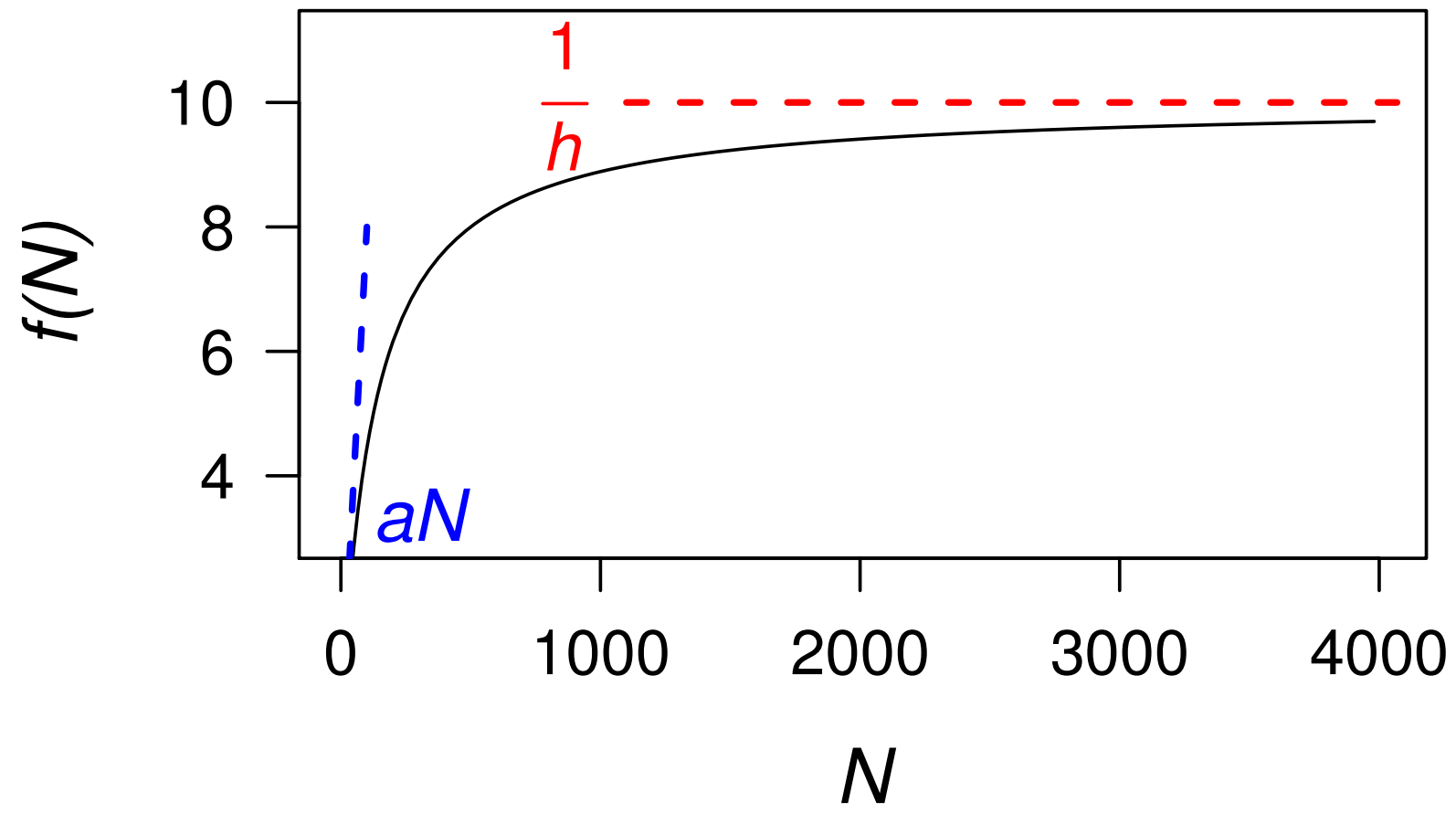

digesting the prey. The attack rate, $a$, describes the space (i.e. area or volume, depending on interaction type; Pawar et al., 2012; Barrios-O'Neill et al., 2016) that a predator searches per unit of time, representing the activity of searching mentioned above. The handling time, $h$, associated with processing the prey, describes the average time that a predator spends on a caught prey item, i.e. subduing, ingesting and digesting. These two parameters also determine the shape of the functional response curve, where the attack rate determines the feeding rate at low prey densities and the handling time determines the maximum feeding rate (Fig. 1)

Figure 1: Schematic curve of type II functional response. The red dashed line denotes the inverse of handling time, $\frac{1}{h}$ (see Eq. (1)) which sets the limit of maximum feeding rate. The blue dashed line denotes the tangent line of the curve at the minimal prey density, $N$ which describes the potential increase of feeding with prey density around low prey densities. This potential increase around low prey densities is determined by the attack rate, $a$ (see Eq. (1)).

Attack rates and handling times indirectly derived through fitting functional response models to feeding data often do not resemble the attack rates and handling times derived by direct observation (Mols et al., 2004; Jeschke and Tollrian, 2005; Sentis et al., 2013; but see Tully et al., 2005, Hauzy et al., 2010). As there are more activities than 'searching 
for prey' and 'subduing the prey' in the life histories or even diurnal cycles of predators (e.g. active and resting periods), a plethora of biological (i.e. physiological and behavioral) processes are collapsed into the attack rate and the handling time (Jeschke et al., 2002; Jeschke and Tollrian, 2005; Casas and McCauley, 2012). Even in a predator's activity period it may not spend the whole time on foraging. For example, grazing ruminants feed in a discrete fashion rather than continuous grazing, i.e. they switch between grazing and resting (Gregorini et al., 2006). As Holling's (1959b) disc equation does not have any term accounting for other activities, e.g. rest or sleeping, handling times and attack rates have to incorporate those time budgets in cases where these other activities apply. Parameter estimates in a long-term functional response experiment are therefore much more likely to incorporate non-foraging behaviours than estimates derived from a shortterm experiment using the same predator-prey pair. Specifically, the feeding rates derived from the long-term study would be lower than those from the short-term study (on the condition that no change in life history traits, e.g. moults). Lower feeding rates will likely affect functional response parameter estimation, decreasing the attack rate and increasing handling time estimates (Fig. $2 \mathrm{a}$ and $2 \mathrm{~b}$ ). Mathematically, the feeding rate, $f(N)$ is negatively related to the handling time, $h$ (Fig. 1) and the increased handling times in long-term experiments (where feeding rate should be lower) is expected for this reason. As the attack rate accounts for the average successful search rate for the entire experimental duration, increasing experimental duration which generally includes more time for other activities than foraging, would lead to reduced attack rates (Casas and McCauley, 2012).

Foraging motivation is also expected to be influenced by predator satiation Jeschke, 2007). Essington et al. (2000) separate the effect of predator satiation to act on two temporal scales: 1) instantaneous satiation occurs when feeding rate exceeds gut capacity 
(constraint of gut size) and 2) integrated satiation occurs when feeding rate exceeds the time required to digest prey (constraint of digestion rate). The longer handling times associated with satiated predators may mostly reflect the constraint of digestion rate, and the comparably shorter handling times of starved predators may be caused by a lack of constraint from gut size. In extreme cases, a predator with a fully-filled gut will be unable to feed even with infinite food supply, a well-known phenomenon called 'digestive limit' (Kleiber, 1961; Herbers, 1981). It describes a phenomenon that consumers are able to fill-up their guts and meet their energy requests rather easily, e.g. on a daily basis (Jeschke, 2007). According to this physiological phenomenon, gut sizes and digestion rates could be limiting factors for the resulting (maximum) feeding rates and associated handling times in the functional response models (Jeschke et al., 2002, 2006). 'Digestive limits' have been demonstrated in a range of vertebrate species but only few invertebrates (Karasov and McWilliams, 2005; Jeschke and Tollrian, 2005; Jeschke, 2007). Under the assumption that digestive limits are a rather general mechanism holding for most consumers, the satiation level of a predator before a feeding study will influence the estimate of handling time (Anderson et al., 1978; Jeschke et al., 2002; Jeschke, 2007). Testing pre-fed predators in feeding trials would then lead to longer handling times compared to testing starved ones. The time budgets of the handling time of a satiated predator would involve not only the time for killing $\left(t_{k i l l}\right)$ and ingesting $\left(t_{i n g}\right)$, but also the time for digestion $\left(t_{d i g}\right)$ and even time for other activities (see Fig. 22.)

As the experimental duration increases, the probability of reaching satiation would increase systematically for every efficiently foraging predator. Thereafter, if the experimental duration is long enough and prey is sufficient, the predator can reach satiation and the constraint of digestive limit is very strong. In this case, the handling time could be influenced by including the time budget for digestion. As the experimental duration 


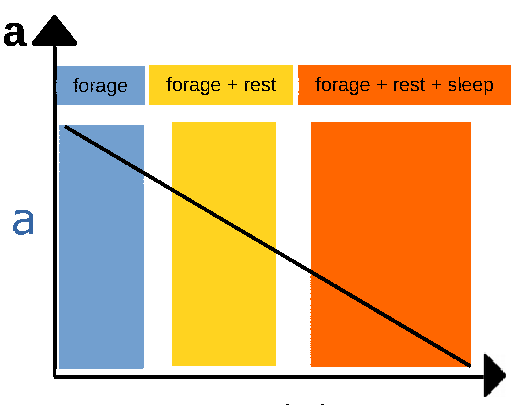

Experimental duration

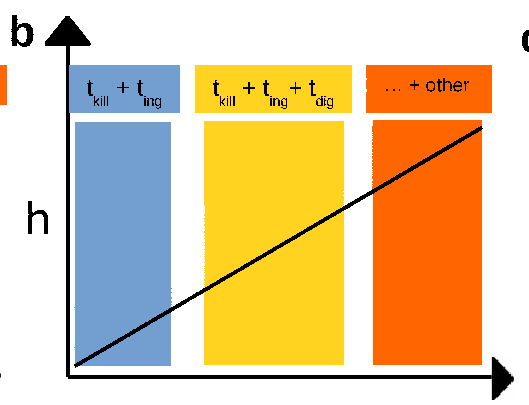

Experimental duration

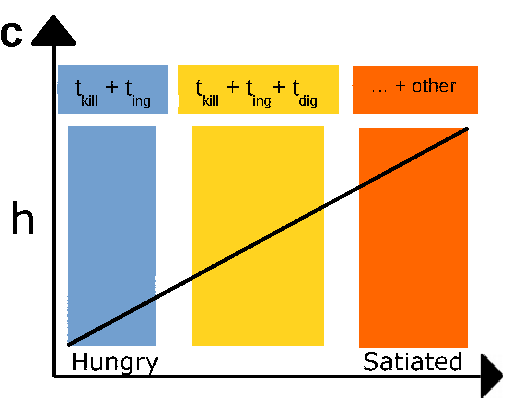

Predator satiation level

Figure 2: The potential effects of experimental duration (panel $\mathbf{a}$ and $\mathbf{b}$ ) and satiation level of the predator (panel c) on functional response parameter estimates. We hypothesized that increasing experimental duration would lead to decreasing attack rates, $a$ (Eq. (1), panel a). Elongated experiments may lead to increased handling times, $h$ (Eq. (1), panel b). We also hypothesized that a satiated (pre-fed) predator shall result in longer handling times $(h)$ than hungry (starved) predators (panel $\mathbf{c}$ ). The text "other" in panels $\mathbf{b}$ and $\mathbf{c}$ denotes time spent on non-feeding activities, e.g. sleep.

further increases, other activities of the predator (e.g. sleep) could be involved. In this case, elongated experimental duration can additionally increase handling time by incorporating a growing proportion of non-feeding activities.

For this study, we used a data set from Rall and colleagues (2012) and updated it with information on starvation and experimental time. We focused on type II functional responses leaving 451 distinct data points from 61 peer-reviewed publications. The data mostly consists of controlled laboratory experiments (99\%) with arthropods (78\%) and vertebrates $(17 \%)$ as predators. Prior to our analyses we hypothesized that, 1) experimental duration has systematic effects on functional response parameters, particularly on the attack rate, and that, 2) the influence of predator satiation on handling time holds over a wide range of different taxa, body masses, and dimensionality of consumer search space. As elaborated above, we assume that in general, satiated predators should consume fewer prey than hungry ones on the premise that all other conditions are the same. Therefore, 3) the handling time of satiated predators should be longer than that of hungry ones as it should incorporate additional time budgets for digestion and activities unrelated to 
foraging.

\section{Methods}

\section{Data and statistical analysis}

We analyzed a data set of published functional responses from empirical studies (Rall et al., 2012). To be included in our analyses studies needed to report experimental duration, consumer and resource body masses, as well as experimental temperatures, as these are main drivers for functional response parameter estimates (Hansen et al., 1997; Rall et al., 2012, Kalinkat et al., 2013; Kalinkat and Rall, 2015). Additionally, we checked and included information on the satiation levels of predators. The predator satiation is represented by "feeding-or-not" prior to the studies, i.e. 'fed' for the predators which were fed before the feeding trials and 'starved' for the predators which were isolated from food source before the feeding trials.

In order to assemble the data set we excluded functional responses derived from experiments which 1) lacked information on experimental duration or predator satiation levels, 2) were not type-II functional responses and 3) excluded ones that are for parasitoids (not suitable for testing predator satiation). The final data set consisted of 451 functional responses from 61 studies (see the full bibliography in Appendix I). It spans 14 orders of magnitude of predator body-mass and covers predator species from 28 taxonomic orders (see Appendix I). It includes 338 and 113 functional responses for starved and fed predators, respectively, and it includes data on experimental duration ranging from $0.08 \mathrm{~h}$ to $240 \mathrm{~h}$ in which $67.6 \%$ are exactly $24 \mathrm{~h}$. It also includes functional responses for studies performed in two- and three-dimensional spaces, in which 243 were two-dimensional interactions 
and 208 were three-dimensional interactions. We chose to also analyze dimensionality as the units of attack rates are different in two- and three-dimensional spaces (i.e. $\left[\mathrm{m}^{2} \mathrm{~s}^{-1}\right]$ and $\left.\left[\mathrm{m}^{3} \mathrm{~s}^{-1}\right]\right)$ which might also cause varying scaling relationships (Pawar et al., 2012; Barrios-O'Neill et al., 2016).

In the following steps, we analyzed the functional response parameters attack rate, $a\left[\mathrm{~m}^{2} \mathrm{~s}^{-1} \mid \mathrm{m}^{3} \mathrm{~s}^{-1}\right]$, and handling time, $h[\mathrm{~s}]$ in relation to experimental duration, $t_{e}[\mathrm{~s}]$ and predator satiation, $S$ (starved, $S_{y}$ or fed, $S_{n}$ ). To account for strong effects of predator body mass $(M[\mathrm{mg}])$, temperature $(T[\mathrm{~K}])$ and dimensionality $(2 D / 3 D)$ we also added these as explanatory variables (Rall et al., 2012, Pawar et al., 2012). Prior to the analysis we ln-transformed the explanatory variables body mass and experimental duration and applied an Arrhenoius transformation to temperature $\left(\frac{T-T_{0}}{k T T_{0}}\right)$, where $k\left[\mathrm{eV} \mathrm{K}^{-1}\right]$ is the Boltzmann's constant and $T_{0}\left(293.15 \mathrm{~K}, 20^{\circ} \mathrm{C}\right)$ is the normalization temperature. Therefore, the intercepts of temperature scalings are shifted to the values at normalization temperature (for more details see Gillooly et al., 2001; Rall et al., 2012). We also tested the collinearity between independent variables, a test on variance inflation factor (VIF) (Zuur et al., 2010). This test was operated in R (R Core Team, 2016) using the 'corvif' function provided by Zuur et al. (2009). According to Zuur et al. (2010), the independent variables of a linear mixed-effects model shall all have the values of VIF less than 3 to make sure that there are not correlation between them (i.e. multicollinearity). The result showed that there is no collinearity between any independent variables, the VIF of predator mass 1.64, temperature 1.25, experimental duration 1.33, predator satiation 2.19 and dimensionality 2.18 .

Thereafter, we used Bayesian Information Criterion (BIC; Zuur et al., 2009, p. 121) to select the optimal random structures of the linear models which were fitted according to restricted maximum likelihood ('REML' Pinheiro et al., 2016, for more details see 
222

22

Appendix II). We analyzed the data with linear mixed-effects models ('lme' function in 'nlme' package in R; Pinheiro et al., 2016, R Core Team, 2016). We included all pairwise interactions of the fixed variables while selecting the optimal random structure for the models of both attack rate and handling time (these full models see Appendix II). After selecting the optimal random effects structure, the BIC values for attack rate and handling time models were computed using the 'dredge' function in the "MuMIn" package in $\mathrm{R}$ (Bartoń, 2016). Optimal models were then selected according to the lowest BIC value following Raftery (1995). Accordingly, $\triangle \mathrm{BIC}$ for each second best fitting model should be at least $>2(\Delta \mathrm{BIC}=\mathrm{BIC}-\min (\mathrm{BIC}))$.

217

\section{Results}

We first selected the appropriate models based on their $\triangle \mathrm{BIC}$ for both, attack rate $(\Delta \mathrm{BIC}$ for second best model $=15.89)$ and handling time $(\Delta \mathrm{BIC}=7.98)$. The selected model showed that attack rate can be described by predator body mass, temperature, experimental duration and dimensionality (Tab. 1):

$$
\begin{aligned}
& \ln \left(a_{2 D}\right)=\ln \left(a_{0_{2 D}}\right)+b \ln (M)+E_{a} \frac{T-T_{0}}{k T T_{0}}+i \ln \left(t_{e}\right) \\
& \ln \left(a_{3 D}\right)=\ln \left(a_{0_{3 D}}\right)+b \ln (M)+E_{a} \frac{T-T_{0}}{k T T_{0}}+i \ln \left(t_{e}\right)
\end{aligned}
$$

\section{Handling time can be described by predator body mass, temperature and predator satiation} (Tab. 1): 
225

$$
\begin{aligned}
& \ln \left(h_{S_{y}}\right)=\ln \left(h_{0_{S_{y}}}\right)+c \ln (M)+E_{h} \frac{T-T_{0}}{k T T_{0}} \\
& \ln \left(h_{S_{n}}\right)=\ln \left(h_{0_{S_{n}}}\right)+c \ln (M)+E_{h} \frac{T-T_{0}}{k T T_{0}}
\end{aligned}
$$

Table 1: Statistical results for attack rate and handling time. All interaction terms have been excluded by model selection (see Methods for details).

\begin{tabular}{llllll}
\hline & Variable & & Estimate & S.E. & p-value \\
\hline \multirow{4}{*}{ attack rate } & dimension & $\ln \left(a_{0_{2 D}}\right)$ & -10.59 & 1.96 & $<0.01$ \\
& predator mass & $\ln \left(a_{0_{3 D}}\right)$ & -12.65 & 1.93 & $<0.01$ \\
\cline { 2 - 6 } & temperature & $E_{a}$ & 0.49 & 0.08 & $<0.01$ \\
& experimental duration & $i$ & -0.56 & 0.06 & $<0.01$ \\
& predator satiation & excluded & & & \\
\cline { 2 - 6 } handling time & predator satiation & $\ln \left(h_{0_{S_{y}}}\right)$ & 10.64 & 0.44 & $<0.01$ \\
& predator mass & $\ln \left(h_{0_{S_{n}}}\right)$ & 13.01 & 0.73 & $<0.01$ \\
\cline { 2 - 6 } & temperature & $E_{h}$ & -0.73 & 0.05 & $<0.01$ \\
& experimental duration & excluded & & 0.10 & $<0.01$ \\
& & & & & \\
\hline
\end{tabular}

In equations above (Eqs. (2), (3)), $a_{0}$ and $h_{0}$ are constants, $b$ and $c$ are the scaling exponents for predator body mass, $M[\mathrm{mg}], E_{a}$ and $E_{h}[\mathrm{eV}]$ are activation energies describing the exponents of temperature and $i$ represents the scaling exponent of attack rate for experimental duration. The attack rate scaled negatively with experimental duration and its model included the influence of dimensionality on its intercepts (panel A, $\mathrm{B}$ and $\mathrm{C}$ of Fig. 3, Tab. 1). The handling time included the effect of predator satiation level, resulting in different constants for starved and fed predators, respectively (panel D and E of Fig. 3, Tab. 11.

${ }^{a}$ see Eq. 22, (3) 

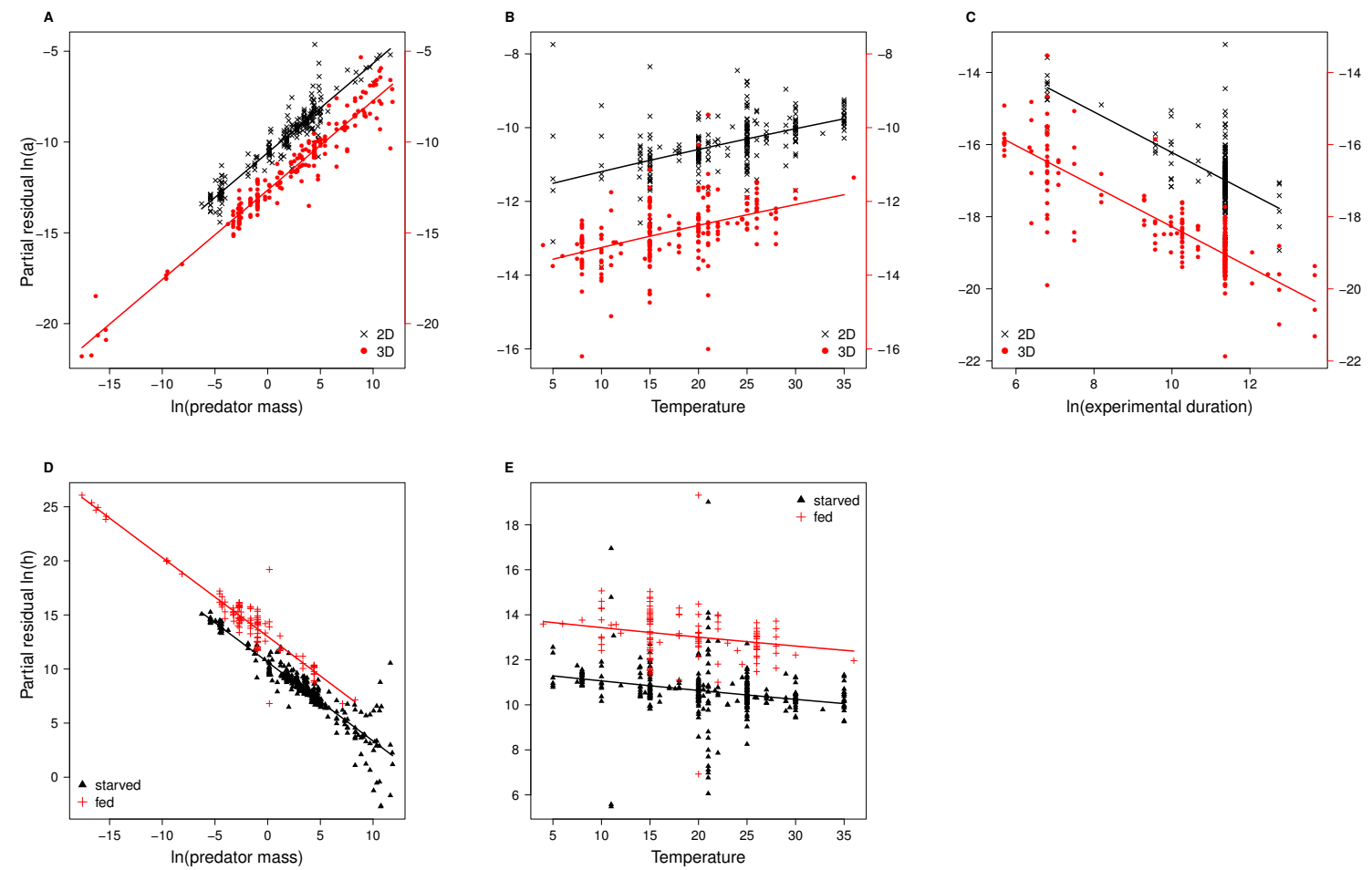

Figure 3: Dependencies of attack rate and handling time. Partial residuals are plotted on the y-axes and all variables other than temperature were ln-transformed. (For details on the derivation of partial residuals see Appendix II). The attack rate $\left(a,\left[\mathrm{~m}^{2} \mathrm{~s}^{-1} \mid \mathrm{m}^{3} \mathrm{~s}^{-1}\right]\right)$ increases with predator body mass $[\mathrm{mg}]$ (panel A), temperature $\left[{ }^{\circ} \mathrm{C}\right]$ (panel B), and decreases with experimental duration [s] (panel C; see Tab. 1). Data of attack rates in two- and three-dimensional cases are plotted with different colors as they have different units (see legends). Handling time $(h)$ decreases with predator body mass (panels D) and temperature (panels E), while handling times for fed predators are longer than those for starved predators (see legends and Tab. 1). 
234

\section{Discussion}

Here we used a large data set of empirical functional responses to investigate if the experimental duration and satiation level of predators have systematic effects on the estimates of functional response parameters. We included studies where feeding data were fitted to the most widespread 'type II functional response' model. Notably, the resulting data set contains predator-prey pairs from a wide variety of ecosystem types including the marine, freshwater and terrestrial realm, as well as a wide range of taxa, from protists to vertebrates. Our results demonstrate that attack rate estimates decrease with increasing experimental duration and that the handling times of satiated predators are longer than those of hungry predators. Thus, two of our hypotheses were supported by our findings (Fig. 22 , c), whereas our hypothesis that increasing experimental duration increases handling time was not supported (Fig. 2b).

Our analyses of attack rates illustrated the influence of predator body mass, temperature and experimental duration. While the results of the effects of predator body mass and temperature on attack rates are consistent with previous studies (Rall et al., 2012; Fussmann et al., 2014) we show here that there is a general effect of experimental duration on the estimates of attack rates that holds across a wide range of taxa and body masses. The finding that attack rate decreases with experimental duration is intuitive to understand and can be attributed to biological mechanisms (Jeschke et al., 2002, Jeschke, 2007). In general, it suggests that the shorter the experimental duration relative to the generation time of the predators, the greater the potential for laboratory experiments to miss important time constraints on foraging. Longer experimental durations will automatically involve a higher proportion of non-feeding activities in foraging experiments. Within a diurnal cycle (24 hour period), the majority of 'other activities' consists of resting and sleeping for most animals (Campbell and Tobler, 1984). Therefore, assuming 
all other conditions are kept constant (e.g. identical predator-prey pair with constant size ratios, identical and standardized satiation levels of the predators), the attack rate estimates derived from a feeding study of 24 hours will be smaller than those obtained through a short-term experiment that includes only the high-activity window out of the diurnal cycle of a given predator (Casas and McCauley, 2012). Despite the suggestion that gut sizes of some predators can be somewhat phenotypically plastic (over periods of weeks or months; Karasov and McWilliams, 2005; Van Gils et al., 2005), we still found that the handling times for satiated predators are longer than those for starved predators. If the gut plasticity plays a big role, we shall have found the opposite, i.e. the starved predators would have had reduced gut capacities and thus showed longer handling times. $96 \%$ of the data used in this study are from experiments within 24 hours. Therefore our findings on the effects of experimental duration are not confounded by changes in gut capacity (Fig. 2a). In addition, if a functional response experiment lasts for the whole generation time of the predator, the resulting functional response parameters are supposed to be a good match to those estimated from long-term population dynamics, e.g. experiments with protists (DeLong et al., 2014).

There are a few empirical case studies also supporting our findings relating to the effects of experimental duration. For instance, Fox and Murdoch (1978) tested how functional responses of a predatory water bugs (Notonecta hoffmanni) vary between short-term (3 hours) and long- term (12 hours) experiments (Fox and Murdoch, 1978). Even though Fox and Murdoch (1978) did not perform a statistical analysis to compare the estimates of functional response parameters between short- and long-term experiments, the estimated values for attack rates are consistent with our results. Another recent modeling study confirmed this effect of experimental duration on the estimates of attack rates and explicitly highlighted that the inclusion of different activities during diurnal cycles may 
bias attack rate estimation (Casas and McCauley, 2012). Our results indicate that these findings of Casas and McCauley (2012) are likely generalizable to most predator-prey pairs. For future studies, it would also be important to address how longer feeding trials, over several weeks or even months, will affect the estimation of interaction strengths (Buckel and Stoner, 2000).

Our results did not show an effect of predator satiation level on attack rates. Jeschke (2007) mentioned that one way to affect the attack rate is through affecting foraging motivation of the predators (Barrios-O'Neill et al., 2016). The foraging motivation of the predators, in turn, can be affected by stressors or distractors on predators, e.g. threat from a top-predator. We can only speculate that in the environments of most functional response experiments, there are no such external stressors on predators to affect their foraging motivations (but see Toscano and Griffen, 2014; Wasserman et al., 2016). Therefore, we might expect significant effects of predator satiation on foraging motivation only if external stressors like top-predators enhance the differences.

Our statistical results documented systematic influence of predator body mass, temperature and predator satiation levels on handling times. Notably, experimental duration had no effect on handling times. With a data set that includes both invertebrate and vertebrate predators, we showed that the estimates of handling times for starved predators were lower than those for the fed ones. Previous studies suggested the influence of satiation level on handling times mostly for vertebrate predators (Karasov and McWilliams, 2005, Jeschke and Tollrian, 2005; Jeschke, 2007). Particularly, Anderson et al. (1978) is one of few experimental studies which explicitly tested how predator satiation level affects the functional response. There, the authors demonstrated that zebra fish (Danio rerio) showed considerably higher maximum feeding rates when they were starved for 24 hours before the experiment compared to satiated fish fed one hour before the trial Anderson 
et al., 1978). Here we generalized this finding to invertebrates, as the majority $78 \%$ of the data we analyzed are from arthropod predators. This supports the theoretical assumption that generally, both vertebrate and invertebrate predators may face digestion limits (see also Jeschke et al., 2002; Jeschke, 2007). In one of the rare experimental studies addressing this issue for invertebrates, however, Maselou et al. (2015) found for a predatory mirid bug (Macrolophus pygmaeus) that the estimates of functional response parameters were not affected by predator satiation. This might be due the specific design where four different treatments of gradually differing starvation levels were tested, while a treatment including fully satiated predators was missing. Moreover, all four functional response curves in this study did not seem to reach full satiation (Maselou et al., 2015). The comparison between satiated and starved predators seems to be important for addressing the effects of predator satiation level on functional response parameter estimates. Another study investigated the influence of predator satiation with data of predatory fish (largemouth bass, Micropterus salmoides; Essington et al., 2000). In agreement with our finding, the authors state that feeding rates are reduced by predator satiation (Essington et al., 2000$)$.

To better address the effect of predator satiation on functional response parameters in future studies, we shall keep in mind the effect of predator satiation acting on two different temporal scales: Essington et al. (2000) suggests separating the effect of predator satiation to act on two temporal scales: 1) instantaneous satiation which reflects the constraint of gut size and 2) integrated satiation which reflects the constraint of digestion rate (Essington et al., 2000) which is in line with suggestions by Jeschke and colleagues (2002; 2006). The longer handling times associated with satiated predators may mostly reflect the constraint of digestion rate, and the comparably shorter handling times of starved predators may be caused by a lack of constraint from gut size. We used binary data of predator satiation rather than the absolute time that the predator was starved 
prior of the experiments due to two reasons: 1) for some data that we had in this dataset, the absolute starvation time are lacking; 2) an alternative analysis with a restricted dataset including only data points where absolute starvation time was available showed similar results than the analysis of the complete dataset with binary data (alternative analysis not shown here). In future studies, a predator's starvation time that is relative to predator body mass or a starvation time that is relative to predator's life span is worthy to be analyzed. These specific starvation time may help to spread the cluster of data points where experimental duration is around 24 hours and give better insight to the question.

Our meta-analysis showed that attack rates of two- and three-dimensional experiments demonstrate the same slope using the ln-transformed linear model. Thus Pawar et al. (2012) found different slopes of attack rates for two- and three-dimensional cases. One obvious difference is that their dataset included not only type II but also type III functional responses. Therefore, Pawar et al. (2012) showed that the attack rates at minimum measured prey densities. This could be one reason why we obtained a different result. Type III functional responses might respond differently to dimensionality than type II functional responses, for instance Type III functional response could indicate a complex spatial structure. Therefore, different dimensionalities together with the complex transition between type II and type III responses might cause these varying responses of attack rates (Barrios-O'Neill et al., 2016; Li et al., 2017). In contrast, our dataset is dominated by data from laboratory functional response experiments where unstructured, artificial habitat prevails (Rall et al., 2012, Kalinkat and Rall, 2015).

Empirical studies that aim to quantify interaction strengths are time-consuming and often need extensive replication to investigate how particular effects drive attack rates, handling times, and other parameters in more complex functional response models (Kalinkat et al., 2013; Barrios-O'Neill et al., 2016). Achieving high replication of long-term 
experiments that are close to natural conditions will most often be logistically infeasible. To that end our study demonstrates that short-term functional response studies will most likely lead to overestimated interaction strengths in models of predator-prey dynamics or food webs. However, our results also demonstrate that this bias can be explained by plausible biological mechanisms. Understanding these mechanism and incorporating them when scaling up from local, short-term, studies to population, community or even ecosystem-level effects holds much promise for a better understanding how species interactions shape communities and ecosystems.

\section{Conclusion}

In the present study, we addressed the systematic effects of two common issues in feeding studies, i.e. how experimental duration and satiation levels of predators affect the parameter estimates in widely applied functional response models. Our study indicates clear and intuitive biological mechanisms affecting the functional response parameters. When models parameterized accordingly are scaled up, these effects will likely modify the estimates of the dynamics and stability of populations, food webs, ecosystems, and, ultimately, biodiversity. Theoretically, both higher attack rates and shorter handling times will strengthen the feeding interactions in population and food-web models. Increasing interaction strengths will generally lead to stronger top-down pressure where stronger predator-prey interactions drive food webs into unstable conditions (Rall et al., 2008). Moreover, for predator-prey systems characterized by cycling dynamics, such strengthening will lead to collapse and the extinction of predator species (Rip and McCann, 2011). This has important implications when realistic predictions to be applied on food-web dynamics are sought. Hence both, empiricists who conduct feeding studies to estimate functional 
response parameters, and theoreticians who try to analyze the dynamics and stability of food webs often parameterized with such empirically-derived parameters should critically take into account these effects. Eventually, this will enable more realistic predictions of population and food-web dynamics which are crucial for understanding the consequences of biodiversity loss (Brose et al., 2017) and will help to bridge lingering gaps between theoretical and empirical ecological research (Jeltsch et al., 2013)

\section{Acknowledgements}

We are grateful to Jonathan Jeschke, Christopher Monk, Daniel Barrios-O'Neill and one anonymous reviewer for their valuable comments and suggestions that helped to improve this manuscript. We further thank Vicky Tröger for her assistance in revising and updating the data set.

\section{Funding}

Funding to Y.L. was provided by the German Science Foundation (DFG) via the research group "FOR 1748 - Networks on Networks". Y.L. and B.C.R. gratefully acknowledge support by the German Centre for Integrative Biodiversity Research (iDiv) Halle-JenaLeipzig funded by the DFG (FZT 118). G.K. was also supported by DFG (KA 3029/2-1). The funding sources had no role in study design, data collection and analysis, decision to publish, or preparation of the manuscript. 
400

\section{Conflict of interest statement}

The authors declare no conflict of interest.

\section{Author contribution statement}

G.K., B.C.R. and Y.L. designed the study; Y.L. analyzed the data in the light of discussion with B.C.R. and G.K.; Y.L. wrote the manuscript with substantial further contributions by B.C.R. and G.K.

\section{Data accessibility statement}

All data will be made available as supporting information should the manuscript be accepted. R-Code for analysis will be available from the authors on request.

\section{References}

Anderson, R. M., Whitfield, P. J., Dobson, A. P. and Keymer, A. E. 1978. Concomitant predation and infection processes: an experimental study. - Journal of Animal Ecology 47(3): 891-911.

Barrios-O'Neill, D., Kelly, R., Dick, J. T. A., Ricciardi, A., MacIsaac, H. J. and Emmerson, M. C. 2016. On the context-dependent scaling of consumer feeding rates. - Ecology Letters 19(6): 668-678.

Bartoń, K. 2016. MuMIn: Multi-Model Inference. - R package version 1.15.6. 
Berlow, E. L., Neutel, A.-M., Cohen, J. E., de Ruiter, P. C., Ebenman, B., Emmerson, M., Fox, J. W., Jansen, V. A. A., Iwan Jones, J., Kokkoris, G. D., Logofet, D. O., McKane, A. J., Montoya, J. M. and Petchey, O. 2004. Interaction strengths in food webs: issues and opportunities. - Journal of Animal Ecology 73(3): 585-598.

Brose, U. 2010. Body-mass constraints on foraging behaviour determine population and food-web dynamics. - Functional Ecology 24(1): 28-34.

Brose, U., Blanchard, J. L., Eklöf, A., Galiana, N., Hartvig, M., R. Hirt, M., Kalinkat, G., Nordström, M. C., O’Gorman, E. J., Rall, B. C., Schneider, F. D., Thébault, E. and Jacob, U. 2017. Predicting the consequences of species loss using size-structured biodiversity approaches. - Biological Reviews 92(2): 684-697.

Brown, J. H., Gillooly, J. F., Allen, A. P., Savage, V. M. and West, G. B. 2004. Toward a metabolic theory of ecology. - Ecology 85(7): 1771-1789.

Buckel, J. A. and Stoner, A. W. 2000. Functional response and switching behavior of young-of-the-year piscivorous bluefish. - Journal of Experimental Marine Biology and Ecology 245(1): 25-41.

Campbell, S. S. and Tobler, I. 1984. Animal sleep: A review of sleep duration across phylogeny. - Neuroscience \& Biobehavioral Reviews 8(3): 269-300.

Casas, J. and McCauley, E. 2012. Daily foraging cycles create overlapping time-scales in functional responses. - Oikos 121(12): 1966-1976.

DeLong, J. P., Hanley, T. C. and Vasseur, D. A. 2014. Predator-prey dynamics and the plasticity of predator body size. - Functional Ecology 28(2): 487-493.

Elton, C. S. 1927. Animal Ecology. - New York, Macmillan Co. 
Essington, T. E., Hodgson, J. R. and Kitchell, J. F. 2000. Role of satiation in the functional response of a piscivore, largemouth bass (Micropterus salmoides). - Canadian Journal of Fisheries and Aquatic Sciences 57(3): 548-556.

Fox, L. R. and Murdoch, W. W. 1978. Effects of feeding history on Short-Term and Long-Term Functional Responses in Notonecta hoffmanni. - Journal of Animal Ecology 47(3): 945-959.

Fussmann, K. E., Schwarzmüller, F., Brose, U., Jousset, A. and Rall, B. C. 2014. Ecological stability in response to warming. - Nature Climate Change 4(3): 206-210.

Gillooly, J. F., Brown, J. H., West, G. B., Savage, V. M. and Charnov, E. L. 2001. Effects of Size and Temperature on Metabolic Rate. - Science 293(5538): 2248-2251.

Gregorini, P., Tamminga, S. and Gunter, S. A. 2006. Behavior and Daily Grazing Patterns of Cattle. - The Professional Animal Scientist 22(3): 201-209.

Hansen, P. J., Bjørnsen, P. K. and Hansen, B. W. 1997. Zooplankton grazing and growth: Scaling within the 2-2,000- $\mu \mathrm{m}$ body size range. - Limnology and Oceanography 42(4): $687-704$.

Hauzy, C., Tully, T., Spataro, T., Paul, G. and Arditi, R. 2010. Spatial heterogeneity and functional response: an experiment in microcosms with varying obstacle densities. Oecologia 163(3): 625-636.

Herbers, J. M. 1981. Time Resources and Laziness in Animals. - Oecologia 49(2): 252-262.

Holling, C. S. 1959a. The components of predation as revealed by a study of small-mammal predation of the European pine sawfly. - The Canadian Entomologist 91(5): 293-320.

— 1959b. Some characteristics of simple types of predation and parasitism. - The Canadian Entomologist 91(7): 385-398. 
Jeltsch, F., Blaum, N., Brose, U., Chipperfield, J. D., Clough, Y., Farwig, N., Geissler, K., Graham, C. H., Grimm, V., Hickler, T., Huth, A., May, F., Meyer, K. M., Pagel, J., Reineking, B., Rillig, M. C., Shea, K., Schurr, F. M., Schröder, B., Tielbörger, K., Weiss, L., Wiegand, K., Wiegand, T., Wirth, C. and Zurell, D. 2013. How can we bring together empiricists and modellers in functional biodiversity research?. - Basic and Applied Ecology 14(2): 93-101.

Jeschke, J. M. 2007. When carnivores are full and lazy. - Oecologia 152(2): 357-364.

Jeschke, J. M., Kopp, M. and Tollrian, R. 2002. Predator functional responses: discriminating between handling and digesting prey. - Ecological Monographs 72(1): $95-112$.

— 2004. Consumer-food systems: why type I functional responses are exclusive to filter feeders. - Biological Reviews 79(2): 337-349.

— 2006. Time and energy constraints: reply to Nolet and Klaassen (2005). - Oikos 114(3): $553-554$.

Jeschke, J. M. and Tollrian, R. 2005. Predicting Herbivore Feeding Times. - Ethology 111(2): 187-206.

Kalinkat, G. and Rall, B. C. 2015. Effects of climate change on the interactions between insect pests and their natural enemies. - In: Björkman, C. and Niemelä, P. (eds.), Climate Change and Insect Pests. CABI, pp. 74-91.

Kalinkat, G., Schneider, F. D., Digel, C., Guill, C., Rall, B. C. and Brose, U. 2013. Body masses, functional responses and predator-prey stability. - Ecology Letters 16(9): $1126-1134$. 
Karasov, W. H. and McWilliams, S. R. 2005. Digestive constraints in mammalian and avian ecology. - In: Stark, J. M. and Wang, T. (eds.), Physiological and ecological adaptations to feeding in vertebrates. Science Publishers, pp. 88-112.

Kleiber, M. 1961. The Fire of Life: An Introduction to Animal Energetics.. - Wiley.

Li, Y., Brose, U., Meyer, K. and Rall, B. C. 2017. How patch size and refuge availability change interaction strength and population dynamics: a combined individual- and population-based modeling experiment. - PeerJ 5: e2993.

Maselou, D., Perdikis, D. and Fantinou, A. 2015. Effect of hunger level on prey consumption and functional response of the predator Macrolophus pygmaeus. - Bulletin of Insectology 68(2): $211-218$.

May, R. M. 1972. Will a large complex system be stable?. - Nature 238(5364): 413-414.

Mols, C. M. M., Oers, K. v., Witjes, L. M. A., Lessells, C. M., Drent, P. J. and Visser, M. E. 2004. Central assumptions of predator-prey models fail in a semi-natural experimental system. - Proceedings of the Royal Society of London B: Biological Sciences 271(Suppl 3): S85-S87.

Oaten, A. and Murdoch, W. W. 1975. Functional Response and Stability in Predator-Prey Systems. - The American Naturalist 109(967): 289-298.

Oksanen, L., Fretwell, S. D., Arruda, J. and Niemela, P. 1981. Exploitation Ecosystems in Gradients of Primary Productivity. - The American Naturalist 118(2): 240-261.

Okuyama, T. 2013. Consequences of variation in foraging success among predators on numerical response. - Ecology and Evolution 3(11): 4039-4043.

Pawar, S., Dell, A. I. and Savage, V. M. 2012. Dimensionality of consumer search space drives trophic interaction strengths. - Nature 486(7404): 485-489. 
Pinheiro, J., Bates, D., DebRoy, S., Sarkar, D. and R Core Team 2016. nlme: Linear and Nonlinear Mixed Effects Models. - R package version 3.1-128.

R Core Team 2016. R: A Language and Environment for Statistical Computing. - R Foundation for Statistical Computing, Vienna, Austria.

Raftery, A. E. 1995. Bayesian Model Selection in Social Research. - Sociological Methodology 25: 111-163.

Rall, B. C., Brose, U., Hartvig, M., Kalinkat, G., Schwarzmuller, F., Vucic-Pestic, O. and Petchey, O. L. 2012. Universal temperature and body-mass scaling of feeding rates. Philosophical Transactions of the Royal Society B: Biological Sciences 367(1605): 29232934.

Rall, B. C., Guill, C. and Brose, U. 2008. Food-web connectance and predator interference dampen the paradox of enrichment. - Oikos 117(2): 202-213.

Rip, J. M. K. and McCann, K. S. 2011. Cross-ecosystem differences in stability and the principle of energy flux: Cross-ecosystem differences in stability. - Ecology Letters 14(8): 733-740.

Schröder, A., Kalinkat, G. and Arlinghaus, R. 2016. Individual variation in functional response parameters is explained by body size but not by behavioural types in a poeciliid fish. - Oecologia 182(4): 1129-1140.

Sentis, A., Hemptinne, J.-L. and Brodeur, J. 2013. Parsing handling time into its components: implications for responses to a temperature gradient. - Ecology 94(8): $1675-1680$.

Solomon, M. E. 1949. The Natural Control of Animal Populations. - Journal of Animal Ecology 18: 1-35. 
Thompson, R. M., Brose, U., Dunne, J. A., Hall, R. O., Hladyz, S., Kitching, R. L., Martinez, N. D., Rantala, H., Romanuk, T. N., Stouffer, D. B. and Tylianakis, J. M. 2012. Food webs: reconciling the structure and function of biodiversity. - Trends in Ecology \& Evolution 27(12): 689-697.

Toscano, B. J. and Griffen, B. D. 2014. Trait-mediated functional responses: predator behavioural type mediates prey consumption. - Journal of Animal Ecology 83(6): $1469-1477$.

Tully, T., Cassey, P. and Ferriere, R. 2005. Functional response: rigorous estimation and sensitivity to genetic variation in prey. - Oikos 111(3): 479-487.

Van Gils, J. A., Dekinga, A., Spaans, B., Vahl, W. K. and Piersma, T. 2005. Digestive bottleneck affects foraging decisions in red knots Calidris canutus. II. patch choice and length of working day. - Journal of Animal Ecology 74(1): 120-130.

Wasserman, R. J., Alexander, M. E., Dalu, T., Ellender, B. R., Kaiser, H. and Weyl, O. L. F. 2016. Using functional responses to quantify interaction effects among predators. Functional Ecology 30(12): 1988-1998.

Zuur, A. F., Ieno, E. N. and Elphick, C. S. 2010. A protocol for data exploration to avoid common statistical problems: Data exploration. - Methods in Ecology and Evolution 1(1): $3-14$.

Zuur, A. F., Ieno, E. N., Walker, N., Saveliev, A. A. and Smith, G. M. 2009. Mixed Effects Models and Extensions in Ecology with R. - Statistics for Biology and Health. Springer New York. 